\title{
ALMOST DISTRIBUTIVE LATTICES
}

\author{
U. MADDANA SWAMY and G. C. RAO \\ (Received 15 January 1980; revised 1 August 1980) \\ Communicated by D. E. Taylor
}

\begin{abstract}
The concept of 'Almost Distributive Lattices' (ADL) is introduced. This class of ADLs includes almost all the existing ring theoretic generalisations of a Boolean ring (algebra) like regular rings, $p$-rings, biregular rings, associate rings, $\boldsymbol{P}_{\mathbf{1}}$-rings, triple systems, etc. This class also includes the class of Baer-Stone semigroups. A one-to-one correspondence is exhibited between the class of relatively complemented ADLs and the class of Almost Boolean Rings analogous to the well-known Stone's correspondence. Many concepts in distributive lattices can be extended to the class of ADLs through its principal ideals which form a distributive lattice with 0 . Subdirect and Sheaf representations of an ADL are obtained.
\end{abstract}

1980 Mathematics subject classification (Amer. Math.Soc.): 06 D 99.

\section{Introduction}

After the axiomatisation of Boole's two valued propositional calculus (Boole (1854)), many generalisations of the class of Boolean algebras (rings) have come to light. Among them regular rings (Von Neumann (1936)), $p$-rings (McCoy and Montgomery (1937)), biregular rings (Arens and Kaplansky (1948)), associate rings (Sussman (1958)) and $P_{1}$-rings (Subrahmanyam (1960a)) are worth mentioning. It is observed, in Maddana Swamy and Manikyamba (to appear), that the class of associate rings coincides with the classes of well known Baer rings and $m$-domain rings (Subrahmanyam (1960b)). Also, it coincides with *-rings (Saracino and Weisfenning (1975)). Further the class of triple systems has been introduced by Subrahmanyam (1963) as a lattice theoretic generalisation of

(- Copyright Australian Mathematical Society 1981

This research is financed by the Council of Scientific and Industrial Research, Rafi Marg, New Delhi, India. 
$\boldsymbol{P}_{1}$-rings. For most of the results that are valid in triple systems the additive semigroup structure in the triple system does not play any role. This motivated us to introduce the class of almost distributive lattices in this paper. An Almost Distributive Lattice (ADL) is an algebra $(R, \vee, \wedge)$ which satisfies all the axioms of a distributive lattice with smallest element 0 except possibly the commutativity of $\vee$ and $\wedge$ and the right distributivity of $\vee$ over $\wedge$. Maddana Swamy (to appear) introduced the concept of a Baer-Stone semigroup as a common abstraction of Baer rings and Stone lattices (semilattices) and this class extensively generalises the class of Boolean algebras. A Baer-Stone semigroup is meant a semigroup $(S, \cdot)$ in which to each $x \in S$ there is a central idempotent $x^{*} \in S$ such that $x^{*} S=\{y \in S \mid x y=0=y x\}$ and, to each $a \in S$, the mapping $x \mapsto\left(a^{*} x, a^{* *} x\right)$ is an isomorphism of $S$ onto the semigroup $a^{*} S \times a^{* *} S$. In all the structures mentioned above one can define two operations $V$ and $\wedge$ from the parental operations such that it becomes an ADL. For example, in a Baer-Stone semigroup $(S, \cdot)$, if we define $a \wedge b=a^{* *} b$ and $a \vee b$ to be the unique element of $S$ such that $a^{* *}(a \vee b)=a$ and $a^{*}(a \vee b)=a^{*} b$, then $(R, \vee, \wedge)$ becomes an ADL.

In $\$ 1$ we give the definition of $\mathrm{ADL}$ and we give some preliminary results in ADLs. In $\$ 2$ we study the centre $S^{* *}$ of a Baer-Stone semigroup $(S, \cdot)$ in a more general set up. In $\$ 3$ we define an Almost Boolean Ring and exhibit a one-to-one correspondence between the relatively complemented ADLs and Almost Boolean Rings, which is analogous to the well-known Stone's correspondence. In $\$ 4$ we introduce the concept of an ideal in an ADL $R$ and prove that the set of all principal ideals of $R$ forms a distributive lattice bounded below, through which almost all existing concepts in distributive lattices can be introduced in ADLs. In $\$ 5$ we prove a subdirect representation theorem for associative ADLs which simplifies many results in ADLs. In $\$ 6$ we represent an ADL as an ADL of global sections of a sheaf of dual dense ADLs.

\section{\$1. Almost distributive lattices}

In this section we give the definition of an Almost Distributive Lattice and we give some basic results for which most of the proofs are straightforward verifications.

Definition 1.1. An algebra $(R, \vee, \wedge, 0)$ of type $(2,2,0)$ is called an Almost Distributive Lattice, abbreviated as ADL, if it satisfies the following axioms.

(L1) $a \vee 0=a$,

(L2) $0 \wedge a=0$, 
(L3) $(a \vee b) \wedge c=(a \wedge c) \vee(b \wedge c)$,

(L4) $a \wedge(b \vee c)=(a \wedge b) \vee(a \wedge c)$,

(L5) $a \vee(b \wedge c)=(a \vee b) \wedge(a \vee c)$,

(L6) $(a \vee b) \wedge b=b$

for all $a, b, c \in R$.

It can be verified, by means of non-trivial examples, that the above axioms L1 through L6 are all independent. The following example shows that every non-empty set be made into an ADL with any arbitrarily preassigned element as its zero.

EXAMPLE 1.2. Let $X$ be a non-empty set. Fix $x_{0} \in X$. For any $x, y \in X$, define $x \wedge y=y, x \vee y=x$ if $x \neq x_{0}, x_{0} \wedge x=x_{0}$ and $x_{0} \vee x=x$. Then $\left(X, \vee, \wedge, x_{0}\right)$ is an ADL with $x_{0}$ as its 0 .

From now onwards by $R$ we mean an $\operatorname{ADL}(R, \vee, \wedge, 0)$ unless otherwise mentioned. Now we give some basic results.

Lemma 1.3. For any $a \in R$, we have

(1) $a \wedge 0=0$

(2) $a \wedge a=a$

(3) $a \vee a=a$;

(4) $0 \vee a=a$.

Lemma 1.4. For any $a, b \in R$, we have

(1) $(a \wedge b) \vee b=b$;

(2) $a \vee(a \wedge b)=a=a \wedge(a \vee b)$

(3) $a \vee(b \wedge a)=a=(a \vee b) \wedge a$.

Proof. (1) is clear. Since $a=a \vee 0=a \vee(0 \wedge b)=(a \vee 0) \wedge(a \vee b)=a$ $\wedge(a \vee b)$, (2) follows from L4 and (2) of the above Lemma. (3) can be proved on similar lines.

COROLlaRY 1.5. For any $a, b \in R$,

(1) $a \vee b=a$ if and only if $a \wedge b=b$;

(2) $a \vee b=b$ if and only if $a \wedge b=a$.

In view of the above corollary, we give the following

Definition 1.6. For any $a, b \in R$, we say that $a$ is less than or equal to $b$ and write $a<b$ if $a \wedge b=a$ or equivalently, $a \vee b=b$. 
It can be easily seen that the above definition ' $<$ ' is a partial order on $R$. In Lemma 1.4, we mentioned the absorption laws that are valid in ADLs in general. Regarding the remaining absorption laws we have the following

THEOREM 1.7. For any $a, b \in R$, the following are equivalent.

(1) $(a \wedge b) \vee a=a$.

(2) $a \wedge(b \vee a)=a$.

(3) $(b \wedge a) \vee b=b$.

(4) $b \wedge(a \vee b)=b$.

(5) $a \wedge b=b \wedge a$.

(6) $a \vee b=b \vee a$.

(7) The supremum of $a$ and $b$ exists in $R$ and equals $a \vee b$.

(8) There exists $x \in R$ such that $a \leqslant x$ and $b \leqslant x$.

(9) The infimum of $a$ and $b$ exists in $R$ and equals $a \wedge b$.

Proof. The equivalence of (1) and (2) as well as that of (3) and (4) follow from (L4). (5) $\Rightarrow(1)$ and (6) $\Rightarrow(2)$ are clear. We prove (1) $\Rightarrow(5)$. Assume (1).

$$
\begin{aligned}
b \wedge a & =b \wedge\{(a \wedge b) \vee a\}=\{b \wedge(a \wedge b)\} \vee(b \wedge a) \\
& =(a \wedge b) \vee\{a \wedge(b \wedge a)\}[\text { since } b \wedge(a \wedge b)=a \wedge b] \\
& =a \wedge\{b \vee(b \wedge a)\}=a \wedge b .
\end{aligned}
$$

Now assume (2). Then

$$
\begin{aligned}
a \vee b & =\{a \wedge(b \vee a)\} \vee\{b \wedge(b \vee a)\}=(a \vee b) \wedge(b \vee a) \\
& =\{(a \vee b) \wedge b\} \vee\{(a \vee b) \wedge a\}=b \vee a .
\end{aligned}
$$

Thus (2) $\Rightarrow(6)$. By interchanging the roles of $a$ and $b$, we get the equivalence of (1) through (6). Assume (6). Since, for any $a, b \in R, a<a \vee b$, by (6), we have $a \vee b$ is an upper bound of $a$ and $b$. Let $c$ be an upper bound of $a$ and $b$. Then $(a \vee b) \wedge c=(a \wedge c) \vee(b \wedge c)=a \vee b$ and hence, $a \vee b$ is the supremum of $a$ and $b .(7) \Rightarrow(8)$ is clear. Now we prove $(8) \Rightarrow(1)$. Assume (8). Then $(a \wedge b) \vee a=(a \wedge b) \vee(a \wedge x)=a \wedge(b \vee x)=a \wedge x=a$. The equivalence of (5) and (9) follows, dually, from that of (6) and (7).

Lemma 1.8. For any $a, b \in R,(a \vee b) \vee a=a \vee b=a \vee(b \vee a)$

Proof. $a \vee b=a \vee\{b \wedge(b \vee a)\}=(a \vee b) \wedge\{a \vee(b \vee a)\}=\{(a \vee b)$ $\wedge a\} \vee[\{(a \vee b) \wedge b\} \vee\{(a \vee b) \wedge a\}]=a \vee(b \vee a)$

For any $a, b, c \in R$, we have $a \wedge c \leqslant c$ and $b \wedge c<c$ and hence the following is a consequence of Theorem 1.7. 
Lemma 1.9. For any $a, b, c \in R,(a \vee b) \wedge c=(b \vee a) \wedge c$.

Now we prove,

LEMMA 1.10. $\wedge$ is associative in $R$.

Proof.

$$
\begin{aligned}
(a \wedge b) \wedge c= & (a \wedge b) \wedge[c \vee\{a \wedge(b \wedge c)\}] \\
= & \{(a \wedge b) \wedge c\} \vee[(a \wedge b) \wedge\{a \wedge(b \wedge c)\}] \\
= & \{(a \wedge b) \wedge c\} \vee\{a \wedge(b \wedge c)\} \\
& {[\text { by Corollary 1.5, since }(a \wedge b) \vee\{a \wedge(b \wedge c)\}} \\
& =a \wedge\{b \vee(b \wedge c)\}=a \wedge b] \\
= & {[\{(a \wedge b) \wedge c\} \vee a] \wedge[\{(a \wedge b) \wedge c\} \vee(b \wedge c)] } \\
= & a \wedge(b \wedge c) .
\end{aligned}
$$

From Lemmas 1.9 and 1.10 , we have the following

LemMa 1.11. For any $a, b, c \in R, a \wedge b \wedge c=b \wedge a \wedge c$.

More generally, we have

LEMmA 1.12. If $a_{1}, \cdots, a_{n}, b \in R$ and $\left(i_{1}, i_{2}, \cdots, i_{n}\right)$ is any permutation of $(1,2, \cdots, n)$, then $a_{i_{1}} \wedge a_{i_{2}} \wedge \cdots \wedge a_{i_{n}} \wedge b=a_{1} \wedge a_{2} \wedge \cdots \wedge a_{n} \wedge b$.

If $\vee$ is right distributive over $\wedge$ in $R$, then, for any $a, b \in R, a=(0 \wedge b) \vee$ $a=a \wedge(b \vee a)$, so that $\vee$ is commutative and hence we have the following

THEOREM 1.13. Let $(R, \vee, \wedge, 0)$ be an $A D L$. Then the following are equivalent.

(1) $(R, \vee, \wedge, 0)$ is a distributive lattice with smallest element 0.

(2) The poset $(R, \leqslant)$ is directed above.

(3) $\vee$ is commutative in $R$.

(4) $\wedge$ is commutative in $R$.

(5) $\vee$ is right distributive over $\wedge$ in $R$.

(6) The relation $\theta=\{(a, b) \in R \times R \mid b \wedge a=a\}$ is antisymmetric.

COROLLARY 1.14. $R$ contains an element 1 such that $a \wedge 1=a$ for all $a \in R$ if and only if $R$ is a bounded distributive lattice, and hence, for any $a \in R$, $\{x \in R \mid x<a\}$ is a bounded distributive lattice with the induced operations. 
In view of the above corollary, we have the following generalised version of Lemma 1.9.

LeMmA 1.15. If $a_{1}, a_{2}, \ldots, a_{n}, b \in R$ and $\left(i_{1}, i_{2}, \ldots, i_{n}\right)$ is any permutation of $(1,2, \ldots, n)$, then $\left(\bigvee_{j=1}^{n} a_{i_{j}}\right) \wedge b=\left(\bigvee_{j=1}^{n} a_{j}\right) \wedge b$.

THEOREM 1.16. If $R$ has a maximal element, then, for any element $a$ of $R$, there exists a maximal element $x \in R$ such that $a<x$ and hence, $R$ is a distributive lattice with 1 if and only if $R$ has a unique maximal element.

Proof. Let $x$ be a maximal element in $R$. Then, for any $a \in R, a \vee x$ is a maximal element and $a \leqslant a \vee x$.

REMARK 1.17. If $a \leqslant b$ and $x \in R$, then $a \wedge x<b \wedge x, x \wedge a<x \wedge b$ and $x \vee a<x \vee b$. But it is not true, in general, that $a<b$ need to imply that $a \vee x<b \vee x$; in fact, $R$ is a distributive lattice with 0 if and only if for any $a, b \in R, a \leqslant b$ implies that $a \vee x \leqslant b \vee x$ for all $x \in R$.

DefINITION 1.18. For any $a, b \in R, a$ is said to be compatible with $b$ (written $a \sim b)$ if $a \wedge b=b \wedge a$ or equivalently, $a \vee b=b \vee a$. A subset $S$ of $R$ is said to be compatible if $a \sim b$ for all $a, b \in S$. By a maximal set we mean a maximal compatible set.

PropositIon 1.19. (1) The relation $\sim$ on $R$ is reflexive and symmetric.

(2) $a \sim b$ whenever $a \leqslant b$ and hence $0 \sim a$ for all $a \in R$.

(3) If $M$ is a maximal set, then $M$ contains 0 and is closed under the operations $\vee$ and $\wedge$ and hence, $(M, \vee, \wedge, 0)$ is distributive lattice with 0 .

(4) If $M$ is a maximal set, then $M$ is an initial segment in $R$; that is, $a \in R$, $b \in M, a \leqslant b$ imply $a \in M$.

REMARK 1.20. $\sim$ is transitive in $R$ if and only if $R$ is distributive lattice with 0 . However, $\sim$ may be transitive among the nonzero elements of $R$ without $R$ being a lattice. For, consider Example 1.2. Since $\sim$ is reflexive, by Zorn's lemma, we have that every ADL $R$ is a set union of its maximal sets.

\section{Amicable sets}

As mentioned in $\S 0$, every Baer-Stone semigroup $S$ is an ADL in which the set $S^{* *}$ of all closed elements is a maximal set and has the property that to each 
$x \in S, x^{* *}$ is the smallest among all $a \in S^{* *}$ such that $a \wedge x=x$. In this section, we study the properties of $S^{* *}$ in a more general context.

Definition 2.1. Let $M$ be a maximal set. Then an element $a$ of $R$ is said to be $M$-amicable if there is a $d \in M$ such that $d \wedge a=a$. If every element of $R$ is $M$-amicable, then we call $M$ an amicable set.

LEMMA 2.2. Let $M$ be a maximal set and $a$ be an $M$-amicable element. Then there exists an element $d \in M$ such that $d \wedge a=a$ and, if $e \sim d$ and $e \wedge a=a$, then $d<e$. Thus, if $M$ is an amicable set, then to each $a \in R$ there exists $a^{0} \in M$ such that, for any $x \in M, x=a^{0}$ if and only if $x \wedge a=a$ and $a \wedge x=x$ and hence, given $a$ and $M$, such $a^{0}$ is unique.

Proof. Since $a$ is $M$-amicable, there exists an element $b \in M$ such that $b \wedge a=a$. Now, write $d=a \wedge b$ then, since $d \leqslant b, d \in M$ and $d \wedge a=$ $b \wedge a=a$. Suppose $e \sim d$ and $e \wedge a=a$. Then $d \wedge e=e \wedge d=e \wedge a \wedge b$ $=a \wedge b=d$ and hence $d \leqslant e$.

TheOREM 2.3. Let $M$ be a maximal set and $a \in R$ be $M$-amicable. Then there exists a maximal set $M^{\prime}$ in $R$ such that $a \in M^{\prime}$ and the lattice $\left(M^{\prime}, \vee, \wedge\right)$ is isomorphic with the lattice $(M, \vee, \wedge)$.

Proof. Let $M^{\prime}=\{x \wedge(a \vee x) \mid x \in M\}$. Then it can be easily verified that $M^{\prime}$ is a maximal set. Since $a$ is $M$-amicable, there is an element $d \in M$ such that $d \wedge a=a$. Then $(a \wedge d) \wedge\{a \vee(a \wedge d)\}=a \wedge d \wedge a=a$ and hence $a \in M^{\prime}$. Also $x \mapsto x \wedge(a \vee x)$ is a lattice isomorphism of $M$ onto $M^{\prime}$.

The following theorem establishes the relation between the maximal sets and amicable sets in $R$.

THEOREM 2.4. Let $M$ be an amicable set and $M^{\prime}$ a maximal set in $R$. Then the correspondence $x \mapsto x^{0}$ is an isomorphism of $\left(M^{\prime}, \vee, \wedge\right)$ onto a sublattice of $(M, \vee, \wedge)$ containing 0 where $x^{0}$ denotes the unique element of $M$ such that $x \wedge x^{0}=x^{0}$ and $x^{0} \wedge x=x$.

Proof. Follows from the fact that, for any $x, y \in R, x^{0}=y^{0}, x \sim y$ implies $x=y$.

Thus we have

THEOREM 2.5. Any two amicable sets in $R$ are isomorphic. 
Definition 2.6. Let $M$ be a maximal set in $R$. An upper bound of $M$ in $R$ is called a unielement of $M$.

Observe that $M$ has at most one unielement and that is in $M$, if it exists.

LEMma 2.7. Let $u$ be a maximal element of $R$. Then $M_{u}=\{x \in R \mid x<u\}$ is $a$ maximal set in $R$ with $u$ as its unielement.

It is not known whether amicable sets in an ADL exists in general and is still under investigation. However, the answer for this question is affirmative in case $R$ contains maximal elements. More precisely, we have the following

THEOREM 2.8. If $R$ has maximal elements, then the amicable sets of $R$ are precisely the maximal sets with unielements.

\section{Almost Boolean rings}

Analogous to the well-known Stone's correspondence between the class of relatively complemented distributive lattices with 0 and the class of Boolean rings, in this section we establish a one-to-one correspondence between relatively complemented ADLs and the class of Almost Boolean Rings which are defined in this section.

In an $\mathrm{ADL}(R, \vee, \wedge, 0)$, by an interval in $R$, we mean the set $[a, b]:=\{x \in$ $R \mid a \leqslant x \leqslant b\}$ for some $a, b \in R$ such that $a<b$. Clearly, every interval in an ADL is again an ADL and hence is a bounded distributive lattice by Theorem 1.13. Now we give the following

Definition 3.1. An $\operatorname{ADL}(R, \vee, \wedge, 0)$ is said to be relatively complemented if every interval in $R$ is a Boolean algebra.

LEMMA 3.2. An $A D L(R, \vee, \wedge, 0)$ is relatively complemented if and only if, given $a, b \in R$, there exists $x \in R$, such that $a \vee b=a \vee x$ and $a \wedge x=0$ and, in this case, $x$ is unique which we denote by $a^{b}$.

REMARK 3.3. Observe that the ADL $\left(X, \vee, \wedge, x_{0}\right)$ in Example 1.2 is relatively complemented in which, for any $x, y \in X, x^{y}=x_{0}$ if $x \neq x_{0}$ and $x_{0}^{y}=y$.

LEMMA 3.4. If $R$ is relatively complemented and $a, b \in R$, then $a^{b}<b$. 
Proof. Since $a \wedge a^{b}=0$, we have $a \sim a^{b}$. Also $a^{b} \vee a=a \vee b$ so that $a^{b}=a^{b} \wedge\left(a^{b} \vee a\right)=a^{b} \wedge(a \vee b)=a^{b} \wedge b$.

Since $a^{b} \wedge b^{a}=a^{b} \wedge b \wedge b^{a}=0$, we have

LEMMA 3.5. If $R$ is relatively complemented and $a, b \in R$, then $a^{b} \vee b^{a}=b^{a}$ $\vee a^{b}$.

LemMA 3.6. Let $R$ be relatively complemented and $a, b, c \in R$. Then we have the following

(1) $(a \wedge c)^{c}=a^{c}$.

(2) $(a \vee b)^{c}=a^{c} \wedge b^{c}$.

(3) $(a \wedge b)^{c}=a^{c} \vee b^{c}$.

(4) $(a \wedge b)^{a \wedge c}=a \wedge b^{c}$ and $(b \wedge a)^{c \wedge a}=b^{c} \wedge a$.

Proof. (1) Since $a \wedge c \wedge a^{c}=0$, we have $(a \wedge c) \vee a^{c}=a^{c} \vee(a \wedge c)=$ $\left(a^{c} \vee a\right) \wedge\left(a^{c} \vee c\right)=(a \vee c) \wedge c=c$ so that $(a \wedge c)^{c}=a^{c}$.

(2) Clearly $(a \vee b) \wedge a^{c} \wedge b^{c}=0$. Now

$$
\begin{aligned}
(a \vee b) \vee\left(a^{c} \wedge b^{c}\right) & =\left\{(a \vee b) \vee a^{c}\right\} \wedge\left\{(a \vee b) \vee b^{c}\right\} \\
& =\{(a \vee b) \vee c\} \wedge\left\{(a \vee b) \vee b^{c}\right\} \\
& =(a \vee b) \vee\left(c \wedge b^{c}\right)=(a \vee b) \vee b^{c} \\
& =\left\{(a \vee b) \vee b^{c}\right\} \wedge\{(a \vee b) \vee c\}=(a \vee b) \vee c
\end{aligned}
$$

(again by Corollary 1.14). Hence $(a \vee b)^{c}=a^{c} \wedge b^{c}$.

(3) Clearly $a \wedge b \wedge\left(a^{c} \vee b^{c}\right)=0$. Now,

$$
\begin{aligned}
(a \wedge b) \vee\left(a^{c} \vee b^{c}\right) & =(a \wedge b) \vee\left\{\left(a^{c} \vee b^{c}\right) \wedge c\right\} \\
& =\left\{(a \wedge b) \vee\left(a^{c} \vee b^{c}\right)\right\} \wedge\{(a \wedge b) \vee c\} \\
& =\left\{\left(a^{c} \vee b^{c}\right) \vee(a \wedge b)\right\} \wedge\{(a \wedge b) \vee c\} \\
& =\left(a^{c} \vee b^{c} \vee a\right) \wedge\left(a^{c} \vee b^{c} \vee b\right) \wedge\{(a \wedge b) \vee c\} \\
& =(a \vee c) \wedge(b \vee c) \wedge\{(a \wedge b) \vee c\} \\
& =\{c \vee(a \wedge b)\} \wedge\{(a \wedge b) \vee c\}=(a \wedge b) \vee c
\end{aligned}
$$

and hence $(a \wedge b)^{c}=a^{c} \vee b^{c}$.

(4) is a routine verification.

It is not known whether the associativity of $V$ in an ADL is a consequence and investigations in this direction are still going on. In view of this, we call an ADL $R$ associative if the operation $\vee$ in $R$ is associative. However, we prove the following

THEOREM 3.7. A relatively complemented ADL $R$ is associative. 
Proof. Let $a, b, c \in R$. Then

$$
\begin{aligned}
(a \vee b) \vee c= & (a \vee b) \vee(a \vee b)^{c} \\
= & \left(a \vee a^{b}\right) \vee\left(a^{c} \wedge b^{c}\right)=a \vee\left\{a^{b} \vee\left(a^{c} \wedge b^{c}\right)\right\} \\
& \left(\text { since the elements } a, a^{b} \text { and } a^{c} \wedge b^{c}\right. \text { are mutually disjoint) } \\
= & a \vee\left\{\left(a^{b} \vee a^{c}\right) \wedge\left(a^{b} \vee b^{c}\right)\right\} \\
= & \left\{a \vee\left(a^{b} \vee a^{c}\right)\right\} \wedge\left\{a \vee\left(b^{c} \vee a^{b}\right)\right\} \\
= & \{a \vee(b \vee c)\} \wedge\left[a \vee\left\{b^{c} \vee\left(a^{b} \wedge b\right)\right\}\right] \\
= & \{a \vee(b \vee c)\} \wedge\left\{a \vee\left(b^{c} \vee a^{b}\right)\right\} \wedge\left\{a \vee\left(b^{c} \vee b\right)\right\} \\
= & \{a \vee(b \vee c)\} \wedge\left\{a \vee\left(b \vee b^{c}\right)\right\}=a \vee(b \vee c) .
\end{aligned}
$$

Definition 3.8. An algebra $(R,+, \cdot, 0)$ of type $(2,2,0)$ is called an Almost Boolean Ring, abbreviated as $\mathrm{ABR}$, for any $a, b, c, d \in R$, it satisfies the following:

$$
\begin{aligned}
& \text { (R1) } a+0=a, \\
& \text { (R2) } a+a=0, \\
& \text { (R3) }(a b) c=a(b c), \\
& \text { (R4) } a^{2}=a, \\
& \text { (R5) } a(b+c)=a b+a c, \\
& \text { (R6) }(a+b) c=a c+b c, \\
& \text { (R7) }\{a+(b+c)\} d=\{(a+b)+c\} d .
\end{aligned}
$$

It can be verified that the above axioms ( $R 1)$ through $(\mathrm{R} 7)$ are independent. We immediately have from Definition 3.8

Lemma 3.9. For any $a, b, c \in R$, we have

(1) $a 0=0 a=0$,

(2) $a b c=b a c$.

Proof. (1) is clear and (2) follows from the fact that, for any $c \in R$, $R_{c}:=\{a c \mid a \in R\}$ is a Boolean ring under the induced operations + and $\cdot$

THEOREM 3.10. Let $(R, \vee, \wedge, 0)$ be a relatively complemented $A D L$. Define a binary operation + on $R$ by $a+b=a^{b} \vee b^{a}$. Then $(R,+, \wedge, 0)$ is an $A B R$. Further, for any $a, b \in R, a \vee b=a+\{b+(a \wedge b)\}$.

Proof. The first assertion of the theorem follows from Lemma 3.6. Now $a+\{b+(a \wedge b)\}=a+\left\{b^{a \wedge b} \vee(a \wedge b)^{b}\right\}=a+a^{b}=a \vee b$. 
Now, we finish this section by proving the converse of the above theorem.

TheOREM 3.11. Let $(R,+, \cdot, 0)$ be an $A B R$. Define the operation $\vee$ on $R$ by $a \vee b=a+(b+a b)$. Then $(R, \vee, \cdot, 0)$ is a relatively complemented $A D L$ in which, for any $a, b \in R, a+b=a^{b} \vee b^{a}$.

Proof. It is routine to verify that $(R, \vee, \cdot, 0)$ is an ADL. Now observe that, for any $a, b \in R, a \vee b=a+b$ whenever $a b=0$. So that $R$ is relatively complemented, in which, for any $a, b \in R, a^{b}=b+a b$. Finally, for any $a, b \in R, a^{b} \vee b^{a}=a^{b}+b^{a}=(b+a b)(a+a b)=(b+a)(a+b)=a+b$.

\section{Ideals}

In this section we introduce the concept of an ideal in an ADL $R$ which coincides with the usual concept of an ideal in a lattice in the case when $R$ is a lattice and we prove that the set of all principal ideals of $R$ is a distributive lattice with smallest element 0 .

Definition 4.1. A nonempty subset $I$ of an ADL $R$ is said to be an ideal of $R$ if for any $a, b \in I$ and $x \in R$, all the elements $a \vee b, x \wedge a$ and $a \wedge x$ belong to $I$. An ideal $I$ of $R$ is called proper if $I \neq R$. A proper ideal $I$ of $R$ is said to be prime if, for any $a, b \in R, a \notin I$ and $b \notin I$ imply that $a \wedge b \notin I$.

An ideal $I$ of $R$ is again an ADL with the induced operations. For any nonempty subset $S$ of $R,(S):=\left\{\left(\bigvee_{i=1}^{n} s_{i}\right) \wedge x \mid s_{i} \in S, x \in R, n\right.$ is a positive integer $\}$ is the smallest ideal of $R$ containing $S$. In particular, for any $x \in R$, $(x):=(\{x\})=\{x \wedge t \mid t \in R\}$. The set $I(R)$ of all ideals of $R$ is closed under arbitrary intersections and contains $R$. Thus $I(R)$ is a complete lattice in which the lub $I \vee J$ of any two ideals $I$ and $J$ of $R$ is $\{x \vee y \mid x \in I$ and $y \in J\}$. Since, for any $x, y \in R,(x) \vee(y)=(x \vee y)$ and $(x) \cap(y)=(x \wedge y)$, the set $P I(R)$ of all principal ideals of $R$ is a sublattice of $I(R)$.

For any subset $S$ of $R, S^{*}:=\{x \in R \mid x \wedge s=0$ for all $s \in S\}$ is an ideal of $R$ and $S^{*}=(S)^{*}$. An ideal $I$ of $R$ is called a direct summand if there exists an ideal $J$ of $R$ such that $I \vee J=R$ and $I \cap J=\{0\}$. In this case $J$ is called the direct complement of $I$ and $R$ is called the direct sum of $I$ and $J$. Then we have the following:

LEMMA 4.2. $R$ has maximal elements if and only if every direct summand is a principal ideal. 
Proof. Let $x$ be a maximal element of $R$ and $I$ a direct summand of $R$. Choose an ideal $J$ of $R$ such that $I \cap J=\{0\}$ and $I \vee J=R$. Hence, $x=y \vee$ $z$ for some $y \in I$ and $z \in J$. We prove $I=(y)$. Let $t \in I$ then $t=x \wedge t=$ $(y \vee z) \wedge t=(y \wedge t) \vee(z \wedge t)=y \wedge t$ so that $t \in(y)$. Conversely, suppose every direct summand is a principal ideal. In particular, $R$ is a principal ideal. Hence $R$ has maximal elements.

The following theorem gives a necessary and sufficient condition for an ADL $R$ to be relatively complemented.

THEOREM 4.3. $R$ is relatively complemented if and only if every principal ideal is $a$ direct summand.

Proof. Let $R$ be relatively complemented and $x \in R$. We prove that $(x)^{*}$ is the direct complement of $(x)$. Clearly, $(x) \cap(x)^{*}=\{0\}$. Let $y \in R$. Then $x^{y} \in(x)^{*}$ and $y=(x \vee y) \wedge y=\left(x \vee x^{y}\right) \wedge y=(x \wedge y) \vee x^{y} \in(x) \vee$ $(x)^{*}$. Conversely, assume that every principal ideal is a direct summand. Let $x, y \in R$. Then there is an ideal $I$ of $R$ such that $(x) \cap I=\{0\}$ and $(x) \vee I=$ $R$. Choose $z \in I$ and $t \in R$ such that $y=(x \wedge t) \vee z$. Clearly $x \wedge z=0$. Now

$$
\begin{aligned}
x \vee z & =x \vee[z \wedge\{(x \wedge t) \vee z\}] \\
& =(x \vee z) \wedge[x \vee\{(x \wedge t) \vee z\}] \\
& =\{(x \vee z) \wedge x\} \vee[\{(x \vee z) \wedge x \wedge t\} \vee z] \\
& =x \vee\{(x \wedge t) \vee z\}=x \vee y .
\end{aligned}
$$

Hence $R$ is relatively complemented.

If $R$ has maximal elements, then every amicable set is isomorphic with the principal ideal lattice $P I(R)$. More explicitly, if $M$ is an amicable set then the mapping $x \mapsto(x)$ is an isomorphism of $M$ onto $P I(R)$. Further, the mapping $I \mapsto\{(x) \mid x \in I\}$ is an isomorphism of $I(R)$ onto the ideal lattice of $P I(R)$ whose restriction to the sapce Spec $R$ of prime ideals of $R$ is a homeomorphism onto the space Spec $P I(R)$ of prime ideals of the distributive lattice $P I(R)$ with usual hull-kernel topologies.

REMARK 4.4. Almost all the lattice theoretic concepts can be introduced in an ADL $R$ through the distributive lattice $P I(R)$. In particular, we have the following.

THEOREM 4.5. Let $(R, \vee, \wedge, 0)$ be an $A D L$. Then the following are equivalent:

(1) $R$ is relatively complemented.

(2) $P I(R)$ is relatively complemented.

(3) Every prime ideal of $R$ is maximal. 
(4) Every prime ideal of $R$ is minimal.

(5) Spec $R$ is a $T_{1}$-space.

(6) Spec $R$ is a $T_{2}$-space.

\section{Subdirect representations}

If $R$ is an ADL, then the map $a \mapsto(x \wedge a)_{x \in R}$ of $R$ into the product $\pi_{x \in R}(x)$ gives a representation for $R$ as a subdirect product of its principal ideals. But, here we can not say anything about the ADLs $(x)$ except that they have maximal elements. In the following, we prove that any ADL in which the operation $V$ is associative can be represented as a subdirect product of ADLs with at most three elements in which every nonzero element is maximal. From this we get the well-known subdirect representation of a distributive lattice with 0 as a subdirect product of two element chains.

Definition 5.1. An ADL $R$ is said to be discrete if every nonzero element is maximal.

Clearly an ADL $R$ is discrete if and only if $a \wedge b=b$ or equivalently $a \vee b=a$ for all $0 \neq a \in R$. Further, every discrete ADL is associative.

For any $a \in R$, define $\theta_{a}=\{(x, y) \in R \times R \mid a \vee x=a \vee y\}$. If $R$ is associative, then $\theta_{a}$ is a congruence relation on $R$. Now we prove the converse in the following

THEOREM 5.2. Let $R$ be an $A D L$. Then the following are equivalent.

(1) $R$ is associative.

(2) $\theta_{a}$ is a congruence relation on $R$ for all $a \in R$.

(3) $R$ is a subdirect product of discrete $A D L s$ each with at most three elements.

Proof. (1) $\Rightarrow(2)$ and (3) $\Rightarrow(1)$ are clear. Now assume (2). For any congruence relation $\alpha$ on $R$ and $x, y, a \in R,(a \vee x, a \vee y) \in \alpha$ if and only if $(x, y) \in \theta_{a}$ $\vee \alpha$. Hence the quotient ADL $R \mid \alpha$ also satisfies (2) for any congruence relation $\alpha$ on $R$. Hence, by Birkhoff's theorem, it is enough if we prove that every subdirectly irreducible ADL satisfying (2) is discrete with at most three elements. Let $R$ be a subdirectly irreducible ADL satisfying (2). Let $\theta$ be the smallest nonzero congruence on $R$ and choose $(x, y) \in \theta$ such that $x \neq y$. For any $t \in R, \theta^{t}:=\{(x, y) \in R \times R \mid t \wedge x=t \wedge y\}$ is a congruence relation on $R$ and $\theta^{t}=\Delta_{R}$ if and only if $t$ is maximal, where $\Delta_{R}$ denotes the equality congruence on $R$. If both $x$ and $y$ are not maximal, then $(x, y) \in \theta^{x} \cap \theta^{y}$ and 
hence $x=y$. Thus we can assume that $x$ is minimal. Let $0 \neq a \in R$. Since $x \wedge a=a$, we have $a \wedge x \neq 0$. If $a$ is not maximal, then $a \wedge x=a \wedge y$ and $(x, y) \in \theta_{a \wedge x}$ so that $x=(a \wedge x) \vee x=(a \wedge y) \vee y=y$. Hence $R$ is discrete. For any nonzero elements $a \neq b \in R, \Delta_{R} \cup\{(a, b),(b, a)\}$ is a nonzero congruence on $R$ and hence, $R$ has at most two nonzero elements.

\section{Sheaf representation}

In this section we represent an ADL $R$ as an ADL of all global sections of a suitable sheaf. The main tool used for this is the following theorem which is due to Maddana Swamy (1974).

THEOREM 6.1. Let $A$ be an algebra and $L=(L, \vee, \wedge)$ a distributive lattice. Let $\mu: L \rightarrow \mathcal{L}(A)$ be antitone. The presheaf $\left(A / \mu(m), \pi_{m, n}, L\right)$ is complete if and only if $\mu$ is an anti-homomorphism and $\{\mu(m) \mid m \in L\}$ is a permutable sublattice of $\mathcal{L}(A)$, that is, for any $m$ and $n \in L, \mu(m \vee n)=\mu(m) \cap \mu(n)$ and $\mu(m \wedge n)$ $=\mu(m) \circ \mu(n)$.

An ADL $R$ with maximal elements is called dual dense if, for any $x, y \in R$, $x \vee y$ is maximal implies that either $x$ is minimal or $y$ is maximal. If $R$ is any ADL with maximal elements, then the bounded distributive lattice $P I(R)$ is dual dense if and only if $R$ is a dual dense. Also, $P I(R)$ is dense if and only if $R$ is dense.

Let $R$ be an ADL. Recall that, for any $a \in R, \theta^{a}=\{(x, y) \in R \times R \mid a \wedge x$ $=a \wedge y\}$ is a congruence relation on $R$. Further, it can be easily seen that the map $(a) \mapsto \theta^{a}$ is an anti-homomorphism of the distributive lattice $P I(R)$ into the structure lattice $\mathcal{L}(R)$ of $R$ and $\theta^{a \wedge b}=\theta^{a} \circ \theta^{b}$. Hence $\left\{\theta^{a} \mid a \in R\right\}$ is a distributive and permutable sublattice of $\mathcal{L}(R)$. Following Maddana Swamy (1974), (a) $\mapsto \theta^{a}$ gives a complete presheaf of ADLs over the topological space $X=$ Spec $R$. Let $(\delta, \pi, X)$ be the corresponding sheaf. Then the stalk $\delta_{p}$ of $(\mathcal{\delta}, \pi, X)$ at any $p \in X$ will be $R \mid \theta_{p}$, where $\theta_{p} \cup \cup_{a \in R-p} \theta^{a}$. Now, it can be easily seen that $P I\left(R \mid \theta_{p}\right)$ is isomorphic with $P I(R) \mid \alpha_{p}$, where $\alpha_{p}$ is the smallest congruence relation on the distributive lattice $P I(R)$ containing the prime filter $P I(R)-(p)$ in a single equivalence class where $(p)=\{(x) \mid x \in p\}$. Hence $P I\left(R \mid \theta_{p}\right)$ is a dual dense bounded distributive lattice, so that $R \mid \theta_{p}$ is a dual dense ADL. Thus, we have the following

THEOREM 6.2. Every $A D L R=(R, \vee, \wedge, 0)$ is isomorphic with an $A D L$ of global sections of a sheaf $(\delta, \pi, X)$ of dual dense $A D L s$ over the locally compact space $X=\operatorname{Spec} R$. If $R$ has maximal elements, then $R$ is isomorphic with the $A D L$ of all global sections of the same sheaf $(\mathcal{S}, \pi, X)$. 
The authors express their sincere thanks to the referee for his valuable comments and suggestions.

\section{References}

R. F. Arens and I. Kaplansky (1948), 'Topological representation of algebras', Trans. Amer. Math. Soc. 63, 457-481.

G. Boole (1854), 'An investigation into the laws of thought', London (Reprinted by Open Court Publishing Co., Chelsea, 1940).

N. H. McCoy and Montgomery (1937), 'A representation of generalised Boolean rings', Duke Math. J. 3, 455-459.

U. Maddana Swamy (1974), Representation of algebras by sections of sheaves (Doctoral Thesis, Department of Mathematics, Andhra University, Waltair, India).

U. Maddana Swamy (1980), 'Baer-Stone semigroups', Semigroup Forum 19, 385-386.

U. Maddana Swamy and P. Manikyamba, 'The equivalence of Baer rings and associate rings', to appear.

D. Saracino and V. Weispfield (1975), Model theory and algebra (Lecture Notes in Mathematics 498, pp. 307-383, Springer-Verlag, Berlin).

N. V. Subrahmanyam (1960a), 'Lattice theory for certain classes of rings', Math. Ann. 139, 275-286.

N. V. Subrahmanyam (1960b), 'Structure theory for generalised Boolean rings', Math. Ann. 141, 297-310.

N. V. Subrahmanyam (1963), 'An extension of Boolean lattice theory', Math. Ann. 151, 332-345.

I. Sussman (1958), 'A generalisation for Boolean rings', Math. Ann. 136, 326-338.

J. Von Neumann (1936), 'On regular rings’, Proc. Nat. Acad. Sci., USA 22, 707-713.

Department of Mathematics

Andhra University

Waltair - 530003 , India 